\title{
Reactor antineutrino flux and spectrum measurement of Daya Bay Experiment
}

\author{
Haoqi Lu* \\ Institute of High Energy physics, China \\ E-mail: luhq@ihep.ac.cn
}

The Daya Bay Reactor Neutrino Experiment utilizes eight functionally identical detectors with large target volume to measure the antineutrino flux emitted from three pairs of powerful nuclear reactors at different baselines. The detectors are placed underground to provide enough shielding against cosmic rays induced backgrounds. The experiment can perform a high-statistics determination of the absolute reactor antineutrino flux and spectrum. It's found that a deviation in the measured positron prompt energy spectrum with the comparison to model predictions. It's observed a local discrepancy in the energy range of 4-6 MeV. The experiment gave the first measurement of individual spectra from ${ }^{235} \mathrm{U}$ and ${ }^{239} \mathrm{Pu}$. This paper will report the latest measurements of antineutrino flux, spectral shape and evolution of these two quantities with nuclear fuel at Daya Bay.

European Physical Society Conference on High Energy Physics - EPS-HEP2019 -

10-17 July, 2019

Ghent, Belgium

\footnotetext{
*Speaker.

†n behalf of the Day Bay Collaboration
} 


\section{Intrdouction}

The neutrino is a fundamental particle [1,2] and its oscillation can be described in a 3-flavor neutrino framework in past experiments.A parameterization of the standard Pontecorvo-MakiNakagawa-Sakata (PMNS) matrix describing the unitary transformation relating the mass and flavor eigenstates, defines the three mixing angles $\left(\theta_{23}, \theta_{12}\right.$, and $\left.\theta_{13}\right)$ and one charge-parity $(\mathrm{CP})$ violating phase [3,4]. $\theta_{12}$ and $\theta_{23}$ is deterimined by different type neutrino experiments. $\theta_{13}$ is oberaved until $2012[5,6]$ by reactor antineutrino experiments because of its small value.

Most reactor antineutrino oscillation experiments measure antineutrino events via the inverse beta decay(IBD) reaction $\bar{v}+p \rightarrow e^{+}+n$. It's characterized by two time correlated events, the prompt signal coming from the production and subsequent annihilation of the positron, and the delayed signal from the capture of the neutron in the liquid scintillator. Daya Bay experiment use $0.1 \%$ gadolinium $(\mathrm{Gd})$-doped liquid scintillator to increase the capture cross section of thermal neutrons on $\mathrm{Gd}$ and reduce the capture time (about $30 \mu \mathrm{s}$ ) to suppress accidental coincidence backgrounds. The experiment has a good capalbility to study the anti-neutrino flux and spectrum from reactors. The following content of this paper mainly focuses on recent results about reactor antineutrino flux and spectrum measurement from Daya Bay experiment.

\section{Daya Bay experiment}

The Daya Bay experiment is designed to explore the unknown value of $\theta_{13}$ by measuring the survival probability of electron antineutrinos from the nuclear reactors in Daya Bay, China. The experiment use the Daya Bay Nuclear Power Plant complex as the antineutrino source, one of the 5 most prolific sources of reactor neutrinos in the world. The Power Plant consists of 6 reactors with 17.4 GW of total thermal power. Use multiple sites (one far experimental hall (EH3) and two near experimental halls $(\mathrm{EH} 1, \mathrm{EH} 2)$ ) to effectively cancel the flux uncertainty by relative measurements. The experiment employs 8 identically designed detectors to decrease detector related errors. The detectors are installed underground with good shielding and enough overburden to reduce the cosmic-ray muon flux. There are 6 detectors were deployed before August. 2012. All 8 detectors were installed by October, 2012.

The main goals of the experiment are do world leading precision in measurement of $\theta_{13}$ and $\left|\Delta m_{e e}^{2}\right|$ oscillation parameters, study the anti-neutrino flux/spectrum,search for sterile neutrinos and other new physics

\section{Detector}

\subsection{Antineutrino detector}

The experiment uses 8 functionally identical antineutrino detectors (2 at EH1, 2 at EH2 and 4 at EH3), which are cylindrical stainless steel vessels(SSV) with a $5 \mathrm{~m}$ diameter and 5 meter height (Fig.1)[7].The antineutrino detectors (ADs) are filled with Gd doped liquid scintillator for antineutrino event detection. Each detector is equiped with 192 8-inch photomultiplier tubes (PMTs). The detector use a three-zone structure, including a Gd-doped liquid scintillator (GdLS) zone, liquid scintillator (LS) zone and mineral oil (MO) zone. The inner region is the primary target volume 


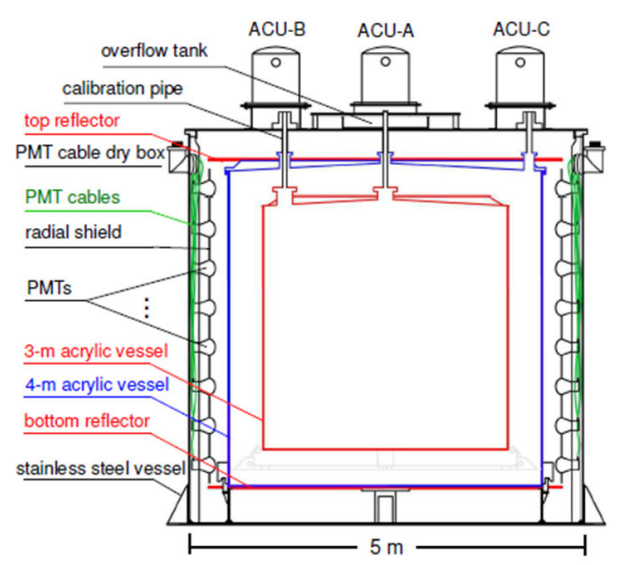

Figure 1: Antineutrino detector of Daya Bay experiment.

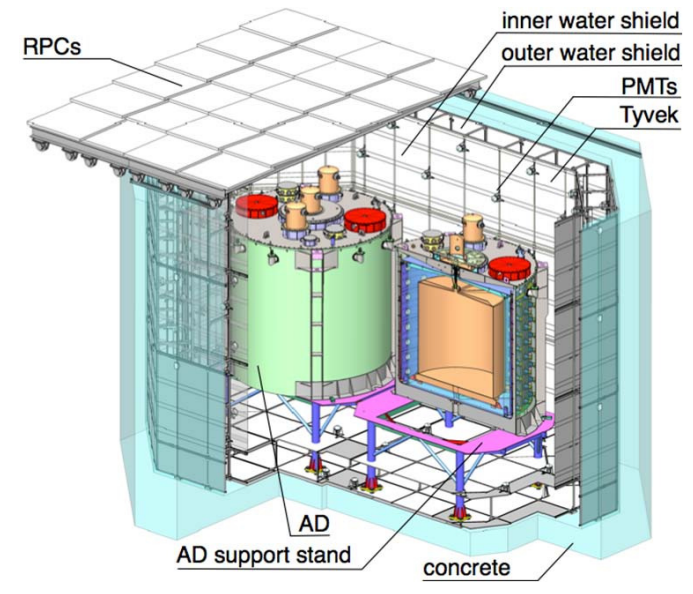

Figure 2: Veto system of Daya Bay experiment. It includes the inner water shield, outer water shield and top RPC detector.

filled with $0.1 \%$ Gd-LS. The middle layer is LS for a gamma catcher, and the outer layer is filled with MO to shielding the radioactivity from outside. Two acrylic tanks(with 3 and 4 meters) are used to separate each layer. Two reflectors at the top and bottom of an AD can improve uniformity and light collection of detector response.

\subsection{Muon veto system}

The Daya Bay muon veto system[8] is shown in Fig. 2 . The ADs are immersed in an octagonal pool with ultrapure water. The pool is divided into outer water shield and inner water shield. The pool is cover with Tyvek sheet. The Tyvek sheet has very high reflectivity $(>95 \%)$ and can increase light collection efficiency.At least $2.5 \mathrm{~m}$ of water surrounds each AD to shield agaist outside radioactivity. There are 288 8-inch PMTs installed in each near hall pool and 384 in the Far Hall. A water circulation and purification system is used in each hall to maintain water quality. The tops of the water Cherenkov detectors are covered by 4 layers of RPCs. The designed efficiency is $>99.5 \%$ with uncertainty less than $0.25 \%$. From muon data analysis, muon detection efficiency of water Cherenkov detector is $>99.7 \%$ for long track muons[7], which is better than the design requirement.

\section{Calibration}

The detector energy calibration is very important to understand the detector energy reponse for particles. The experiment use different natural and artificial sources to calibrate the detector for energy scale, time-variation, non-uniformity and non-linearity.

A lot of calbiration study had been done in the past[7, 9]. We know that the energy nonlinearity of LS is mainly from two sources. One is from scintillator response and the other one is from the readout electronics response. Recently, the experiment did some activities to improve energy nonlinearity. The experiment installated a full Flash ADC(FADC) readout system in EH1 AD1 and take data simultaneously with standard electronics at end of 2015. As the Fig.3 shows, 


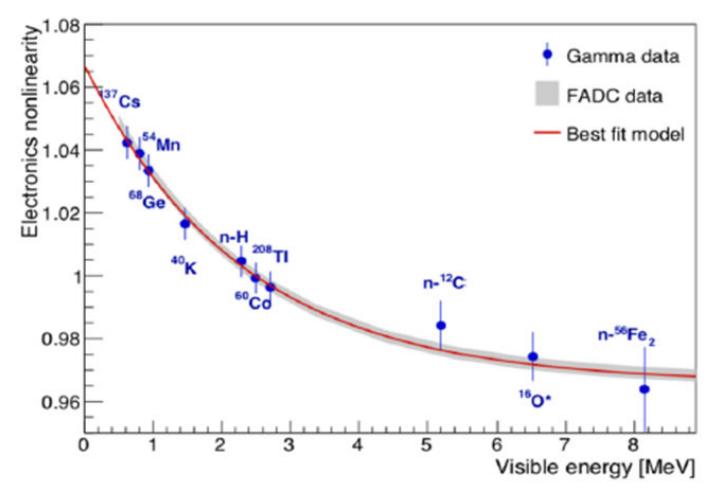

Figure 3: A direct measurement of the electronics non-linearity

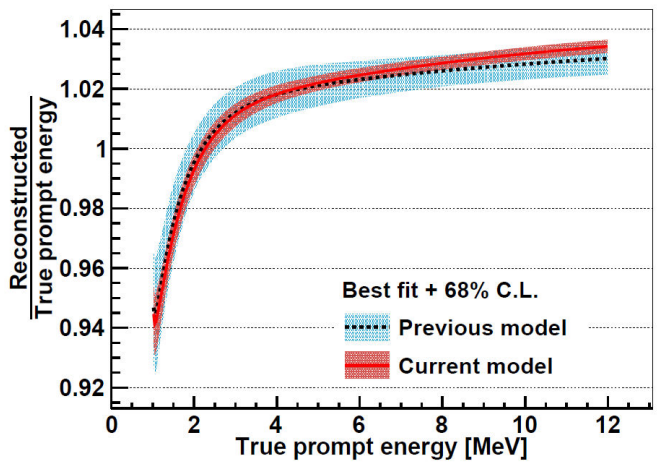

Figure 4: The energy model improvement of the experiment

the experiment has a directly measurement the the electronics non-linearity. The experiment has a very good measuement about the effect.The data compared with gamma data and fit model, they were consist with each other. The experiment also did a special calibration in 2017 . Use ${ }^{60} \mathrm{Co}$ sources with different enclosures to constrain the optical shadowing effect.

A energy model is needed to convert the positron energy to antineutrino energy for the experiment.The experiment bulid a nominal energy model, which is based on fit of mono-energetic gamma lines and ${ }^{12} \mathrm{~B}$ beta-decay spectrum. The figure 4 shows the energy model improvement. Now ,the latest resulsts had reduced the uncertainty from $1 \%$ to $0.5 \%$ since 2018 [10]. It's a big progress about the LS energy response study.

\section{Reactor Antineutrino Flux and Spectrum measurement}

\subsection{Flux}

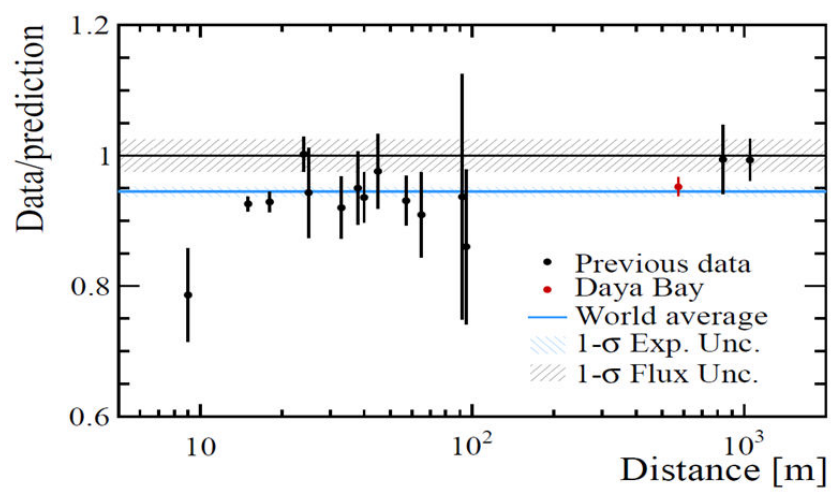

Figure 5: The measured reactor anti-neutrino rate as a function of the distance from the reactor, normalized to the theoretical prediction of Huber+Mueller model. The rate is corrected by 3-flavor neutrino oscillations at the distance of each experiment. The Daya Bay measurement is shown at the flux-weighted baseline (573 $\mathrm{m})$ of the two near halls. 
For the reactor antineutrino flux study, the main uncertainty is from the neutron detection efficiency uncertainty in the past[11]. The experiment do a lot of activities to reduced the uncertainty. We take a lot of new neutron calibration data at different positions of the detector in late 2016 and early 2017. A very detailed MC and data comparison had been done to constrain the neutron detection efficiency uncertainty. Finally, the neutron detection efficiency uncertainty is significantly improved from $1.69 \%$ to $0.74 \%[12]$.

Using 1230 days of data to get the IBD yield is $(5.91 \pm 0.09) \times 10^{-43} \mathrm{~cm}^{2} /$ fission. IBD yield measurement is consistent among 8 ADs. The ratio of measured flux to the predictions is $0.951 \pm 0.014$ (exp) \pm 0.023 (model) $(0.992 \pm 0.015$ (exp. $) \pm 0.027$ (model)) for the Huber+Mueller (ILL+Vogel) model, which is consistent with the global average of previous short baseline experiments(Fig.5).

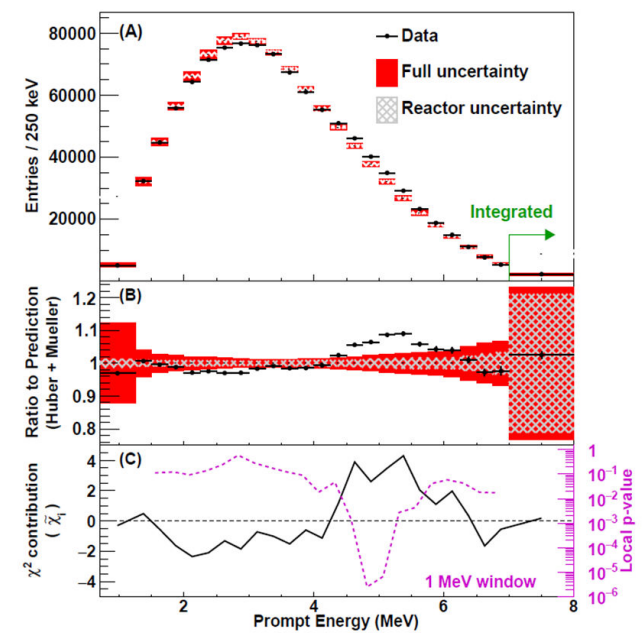

Figure 6: Antineutrino detector of Daya Bay experiment(A) Comparison of predicted(Huber+Mueller) and measured prompt energy spectra. (B) Ratio of the measured prompt energy spectrum to the predicted spectrum (Huber+Mueller model). (C) The defined $\chi^{2}$ distribution of each bin (black solid curve) and local $\mathrm{p}$-values for $1 \mathrm{MeV}$ energy windows (magenta dashed curve).

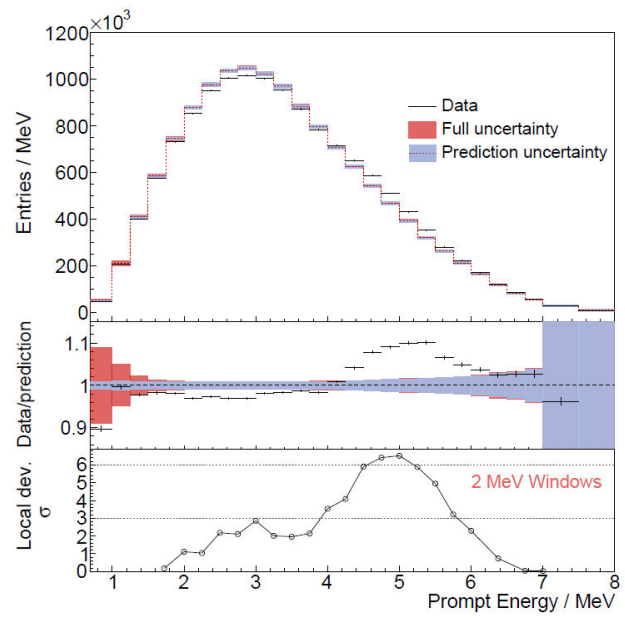

Figure 7: (Top panel) Predicted and measured prompt energy spectra. The prediction is based on the Huber-Mueller model and is normalized to the number of measured events.(Middle panel) Ratio of the measured prompt energy spectrum and the normalized predicted spectrum. The error bars on the data points represent the statistical uncertainty. (Bottom panel) The local significance of the shape deviation in a sliding 2-MeV window showing a maximum $6.3 \sigma$ discrepancy in $4-6 \mathrm{MeV}$.

\subsection{Spectrum}

For the spectrum measurement, using 621 days data with more than 1.2 million IBD candidates, the predicted and measured spectra were compared, and a deviation of $2.9 \sigma$ was found compared to Huber+Mueller model prediction(Fig.6). We found an excess of events in the region of 4-6 MeV with a local significance of $4.4 \sigma[11]$. Excess events characteristics are same as IBD events, correlated with reactor power but time independent. There are no event excess for the spallation ${ }^{12} \mathrm{~B}$ beta spectra at same energy range. It's ruled out detector effects. Recently, the 
experiment have a high-statistics measurement of the spectral shape of reactor anti-neutrinos with 1958 days of data(3.5million IBD events)[14]. The spectrum has a global discrepancy with the Huber+Mueller model prediction at $5.3 \sigma$ and local deviation at $6.3 \sigma$ in 4-6 MeV region(Fig.7).

\section{Reactor antineutrino flux evolution}

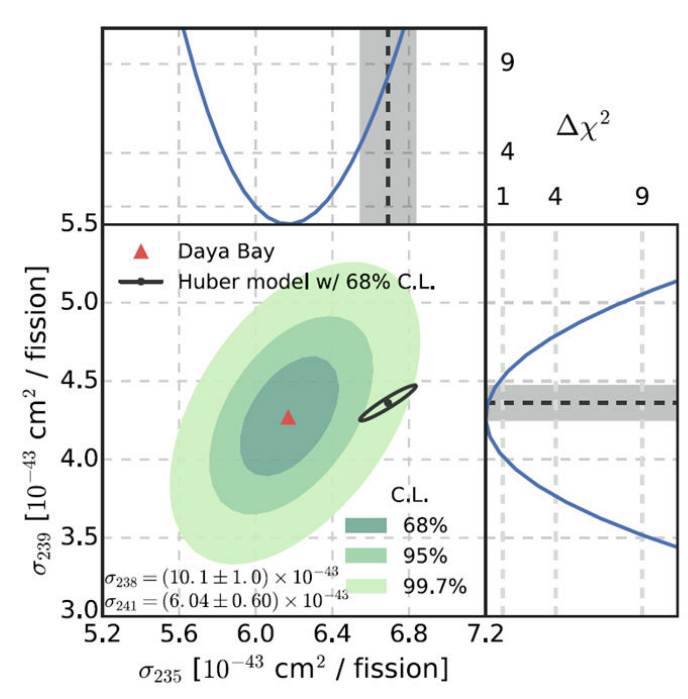

Figure 8: Combined measurement of ${ }^{235} \mathrm{U}$ and ${ }^{239} \mathrm{Pu}$ IBD yields per fission $\sigma_{235}$ and $\sigma_{239}$. The red triangle indicates the best fit of $\sigma_{235}$ and $\sigma_{239}$, while green contours indicate twodimensional $1 \sigma, 2 \sigma$ and $3 \sigma$ allowed regions.

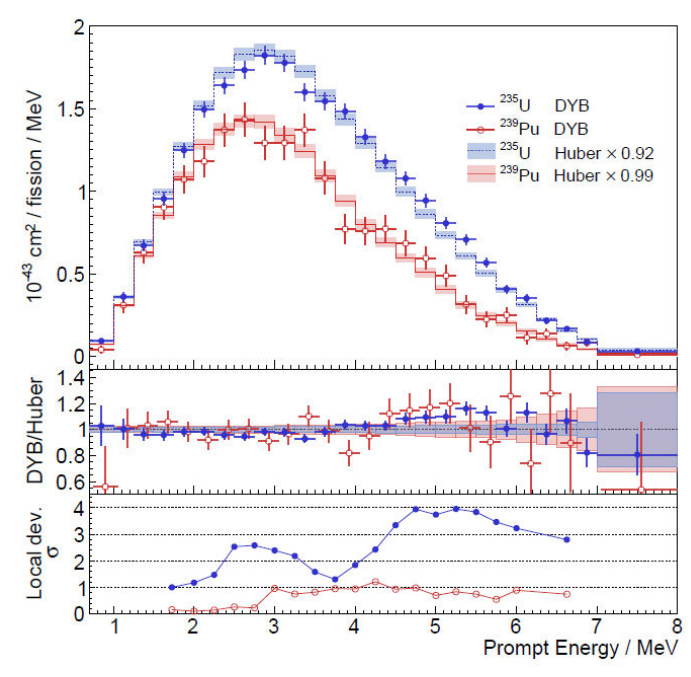

Figure 9: (Top panel) Comparison of the extracted ${ }^{235} \mathrm{U}$ and ${ }^{239} \mathrm{Pu}$ spectra and the corresponding Huber-Mueller model predictions with the normalization factors 0.92 and 0.99 , respectively. The error bars in the data points are the square root of the diagonal terms of the covariance matrix of the extracted spectra. The error bands are the uncertainties from the HuberMueller model. (Middle panel) Ratio of the extracted spectra to the predicted spectra.(Bottom panel) Local significance of the shape deviations for the extracted ${ }^{235} \mathrm{U}$ and ${ }^{239} \mathrm{Pu}$ spectra compared to the model predictions with a sliding 2$\mathrm{MeV}$ window

A 2.2 million IBD candidates(1230 days data set) was used to get the total IBD yield $\bar{\sigma}_{f}$ of $(5.90 \pm 0.13) \times 10^{-43} \mathrm{~cm}^{2} /$ fission with average effective fission fractions F235, F238, F239, and F241. The yield is consist with the previous measurement[11]. It's observed a change in the IBD yield, $d \sigma_{f} / d F_{239}$, of $(-1.86 \pm 0.18) 10^{-43} \mathrm{~cm}^{2} /$ fission over a range of effective ${ }^{239} \mathrm{Pu}$ fission fractions from 0.25 to 0.34 . These yield measurements were used to calculate IBD yield per fission values of $(6.17 \pm 0.17) \times 10^{-43} \mathrm{~cm}^{2} /$ fission and $(4.27 \pm 0.26) \times 10^{-43} \mathrm{~cm}^{2} /$ fission for fits to individual isotopes ${ }^{235} \mathrm{U}$ and ${ }^{239} \mathrm{Pu}$. The calculation is assumed loose (10\%) uncertainties on sub-dominant ${ }^{238} \mathrm{U}$ and ${ }^{241} \mathrm{Pu}$ (central values taken from Huber-Mueller model) as Fig. 8 shown. The measurement of ${ }^{235} \mathrm{U}$ yield is $7.8 \%$ lower than predicted. It's significantly larger than the measurement uncertainty $(2.7 \%)$ [13], which is indicated that overestimated contribution from ${ }^{235} \mathrm{U}$ and it may 
be the primary contributor to the reactor antineutrino anomaly. The new measurements of the ${ }^{235} \mathrm{U}$ antineutrino flux are needed by the future experiment.

\subsection{Individual antineutrino spectra of ${ }^{235} \mathrm{U}$ and ${ }^{239} \mathrm{Pu}$}

The new data set with 1958 days data is used for this analysis. The characteristic of the data is with 3.5 million IBD events and $0.5 \%$ energy scale uncertainty. The data is ordered by ${ }^{239} \mathrm{Pu}$ fission fraction into 20 data groups. The individual spectra of the two dominant isotopes, ${ }^{235} \mathrm{U}$ and ${ }^{239} \mathrm{Pu}$, are extracted using the evolution of the prompt spectrum as a function of the isotope fission fractions. For the not sensitive compents of ${ }^{238} \mathrm{U}$ and ${ }^{241} \mathrm{Pu}$, they were assigned $>10 \%$ uncertainties both on rate and shape as prior inputs. The exract spectrum is shown in Fig 9. It's the first measurement of ${ }^{235} \mathrm{U}$ spectrum and ${ }^{239} \mathrm{Pu}$ spectrum in commercial reactors.In the energy window of 4-6 MeV, a 7\% (9\%) excess of events is observed for the ${ }^{235} \mathrm{U}\left({ }^{239} \mathrm{Pu}\right)$ spectrum compared with the normalized Huber-Mueller model prediction. For IBD yield comparison, the data over prediction of ${ }^{235} \mathrm{U}$ is $0.92 \pm 0.023$ (exp.) \pm 0.021 (model) and $0.99 \pm 0.057$ (exp.) \pm 0.025 (model) for ${ }^{239} \mathrm{Pu}$. Spectral shape local deviation significance is $4 \sigma(4-6 \mathrm{MeV})$ for ${ }^{235} \mathrm{U}$ and $1.2 \sigma$ for ${ }^{239} \mathrm{Pu}$ because of larger uncertainty.

\section{Summary}

The daya Bay expereiment have a good energy non-linearity calibration to improve the uncertainty from $1 \%$ to $0.5 \%$. The experiment measurements the antineutrino flux, which is consistent with previous short baseline experiments. There is a new measurement of reactor antineutrino prompt energy spectrum with 1958 days data. The global discrepancy with the prediction is at 5.3 $\sigma$ and local deviation at $6.3 \sigma$ in $4-6 \mathrm{MeV}$ energy region. Experiment also measure the IBD yield per fission from individual isotopes $\left({ }^{235} \mathrm{U},{ }^{239} \mathrm{Pu},{ }^{238} \mathrm{U},{ }^{241} \mathrm{Pu}\right)$ and found that IBD yield of ${ }^{235} \mathrm{U}$ is $7.8 \%$ lower than prediction. The experiment get the first measurement of ${ }^{235} \mathrm{U}$ and ${ }^{239} \mathrm{Pu}$ spectra in commercial reactors.In the energy window of 4-6 MeV, a $7 \%$ (9\%) excess of events is observed for the $\left.{ }^{235} \mathrm{U}{ }^{(239} \mathrm{Pu}\right)$ spectrum compared with the normalized Huber-Mueller model prediction[14]. The experiment is expected to continue running until 2020. It's expect to get uncertainty in oscillation parameters to below $3 \%$

\section{Acknowledgments}

The article is supported by the National Natural Science Foundation of China (11675203).

\section{References}

[1] C. L. Cowan et al. Science 124, 103 (1956);

[2] F. Reines and C. L. Cowan, Jr., Nature 178, 446 (1956).

[3] B. Pontecorvo, Sov. Phys. JETP 6, 429 (1957) and 26, 984 (1968);

[4] Z. Maki, M. Nakagawa, and S. Sakata, Prog. Theor. Phys. 28, 870 (1962).

[5] Daya Bay Collab. ( F. Anet al.), Phys. Rev. Lett. 108, 171803 (2012). 
[6] RENO Collaboration,Phys. Rev. Lett.108, 191802(2012)

[7] DayaBay Collab. ( F. An et al.),NIM A 685, 78-97 (2012).

[8] DayaBay Collab.(F. An et al.), NIM A 773, 8-20(2015);

[9] Daya Bay Collab. ( F. Anet al.), Phys. Rev. D. 95, 072006 (2017);

[10] Daya Bay Collab.( F. Anet al.), NIM A 940, 230-242(2019)

[11] Daya Bay Collab. ( F. Anet al.), Chinese Physics C, 2017,41(1): 13002-013002

[12] Daya Bay Collab. ( F. Anet al.), arXiv:1808.10836(2018)

[13] Daya Bay Collab. ( F. Anet al.) Phys. Rev. Lett. 118.251801

[14] Daya Bay Collab. ( F. Anet al.),arXiv:1904.07812(2019) 